\title{
The impact of " $4+7$ " volume based drugs procurement on the volume, expenditures, and daily costs of antihypertensive drugs in Shenzhen, China: a interrupted time series analysis
}

\author{
Ying Yang \\ Wuhan University \\ Ruiwen Tong \\ Wuhan University \\ Shicheng Yin \\ Wuhan University \\ Lining Mao \\ Wuhan University \\ Luxinyi Xu \\ Wuhan University \\ Siyu Hao \\ Wuhan University \\ Zongfu Mao ( $\sim$ zfmao@whu.edu.cn ) \\ Wuhan University
}

\section{Research Article}

Keywords: National Centralized Drug Procurement (NCDP), "4+7", volume-based procurement, antihypertensive drugs, China

Posted Date: June 7th, 2021

DOI: https://doi.org/10.21203/rs.3.rs-583026/v1

License: (a) (1) This work is licensed under a Creative Commons Attribution 4.0 International License. Read Full License

Version of Record: A version of this preprint was published at BMC Health Services Research on November 26th, 2021. See the published version at https://doi.org/10.1186/s12913-021-07143-3. 


\section{Abstract}

Background: On January 2019, Chinese government implemented the first round of the National Centralized Drug Procurement (NCDP) pilot of 25 drugs in 4 municipalities and 7 sub-provincial cities in mainland China, referred to as " $4+7$ " policy. In the " $4+7$ " policy, 7 antihypertensive drugs were included. This study was conducted to evaluate the impact of " $4+7$ " policy on the use of policy-related antihypertensive drugs. Method: This study applied single-group Interrupted Time Series (ITS) design. We used drug purchasing data from the Centralized Drug Procurement Survey in Shenzhen 2019, covering 24 months from January 2018 to December 2019. Antihypertensive drugs related to "4+7" policy were selected as study samples, including 7 antihypertensive drugs in the " $4+7$ " List and 17 antihypertensive drugs that have an alternative relationship with the " $4+7$ " List drugs in clinical use. Purchase volume, expenditures, and daily costs were selected as outcome variables, and were measured using Defined Daily Doses (DDDs), Chinese Yuan (CNY), and Defined Daily Drug cost (DDDc), respectively. Segmented linear regression analysis was employed to examine the change of outcome variables before and after the policy intervention. Results: As of December 31, 2019, the completion rate of the agreed purchase volume of the 7 bid-winning antihypertensive drugs reached $173.42 \%$ in Shenzhen. After " $4+7$ " policy, the DDDc of bid-winning antihypertensive drugs significantly decreased by $63.79 \%(-1.30 \mathrm{CNY}, 95 \% \mathrm{Cl}=-1.43$ to -1.18, $\mathrm{p}<0.001)$, while the DDDc of non-winning (0.28 CNY, $95 \% \mathrm{Cl}=0.11$ to $0.46, \mathrm{p}<0.01)$ and alternative $(0.14 \mathrm{CNY}, 95 \% \mathrm{Cl}=0.03$ to $0.25, p<0.05)$ antihypertensive drugs increased markedly. The volume of bid-winning antihypertensive drugs significantly increased by $1311.76 \%$ (3.12 million DDD, 95\% $\mathrm{Cl}=2.14$ to 4.10 , $\mathrm{p}<0.001)$. The overall costs of the seven " $4+7$ " List antihypertensive drugs significantly declined by $101.34 \%$ ( -5.96 million $\mathrm{CNY}, 95 \% \mathrm{Cl}=-7.87$ to $-4.04, \mathrm{p}<0.001)$ after policy intervention, with an absolute reduction of 36.37 million CNY compared with the pre-" $4+7$ " period. Conclusion: An overall satisfying implementation effect was observed in Shenzhen. A preliminary positive policy effect of price cut and cost-saving was observed in the antihypertensive drug category. However, the DDDc of non-winning and alternative drugs increased after policy intervention, suggesting that the price monitoring and drug use management regarding NCDP policy-related drugs should be strengthened. Keywords: National Centralized Drug Procurement (NCDP); "4+7"; volume-based procurement; antihypertensive drugs; China

\section{Introduction}

In China, the total health expenditure has grown rapidly from 145.4 billion Chinese Yuan (CNY) in 2008 to 5799.8 billion CNY in 2018 , with an average compound annual growth rate of $13.4 \%$ [1]. In 2018, the total drug cost was 218.3 billion CNY in China, accounting for $35.8 \%$ of the total health expenditure, which was much higher than the average level of $17 \%$ in the Organization for Economic Co-operation and Development (OECD) countries [2]. It is one of the important reasons for the continuous unreasonable rise of drug expenditures in China that the long-term irregular drug procurement in public medical institutions bred the inflated drug prices [3]. In China, with the increasing aging population, the incidence of hypertension, as well as the overall medication burden of hypertensive patients have increased rapidly year by year [4]. The cardiovascular drug spending accounted for $8.84 \%$ of the total drug spending in China [5]. What's more, the drugs costs of hypertensive patients composed of about $90 \%$ of their outpatient costs [6]. Thus, the price cut and cost saving of antihypertensive drugs is a concern in China. Thus, the price cut and cost saving of antihypertensive drugs have become a noteworthy topic in China.

Worldwide, it is common practices to regulate drug purchase and control drug costs through volume-based drug procurement [7]. In January 2019, the General Office of the State Council of the People's Republic of China (PRC) issued the National Centralized Drug Procurement (NCDP) policy [8]. NCDP is the first policy attempt for volume-based drug procurement at the national level in mainland China. In the first round of NCDP pilot, 11 cities were selected as pilot cities including 4 municipalities (Beijing, Tianjin, Shanghai, Chongqing) and 7 sub-provincial cities (Shenyang, Dalian, Xiamen, Guangzhou, Shenzhen, Chengdu, Xi'an), thus known as the " $4+7$ " policy. In the " $4+7$ " policy, 25 drugs in the " $4+7$ " List were purchased and the pharmaceutical enterprise with the lowest quotation in each drug win the bid by adopting an exclusive bid winning method. Among the 25 bid-winning products, an average price drop of $52 \%$ was emerged with the highest drop of $96 \%$ [9]. The 25 drugs in the " $4+7$ " List involved multiple therapeutic categories, in which 7 antihypertensive drugs were included, i.e., Amlodipine, Irbesartan, Irbesartan hydrochlorothiazide, Fosinopril, Lisinopril, Losartan, Enalapril. The average price reduction was $59.1 \%$ for the 7 bid-winning antihypertensive products. 
Previous studies reported that the implementation of " $4+7$ " policy was generally well progressed in the pilot cities [10-14]. Chen et al. [15] reported an prominent reduction of $41.9 \%$ in the overall costs of " $4+7$ " policy-related drugs in Shenzhen, China after " 4 +7 " policy. Wang et al. [16] found that the drug price index of policy-related drugs significantly decreased after policy intervention. Moreover, the survey in the outpatient of a tertiary hospital found that the implementation of " $4+7$ " policy increased the use of cardiovascular generic drugs and significantly reduced the drug costs of patients [17]. However, previous investigations reminded that the issue of poor efficacy and increased adverse reactions were observed in some " $4+7$ " bid-winning antihypertensive products, which may affect the clinical use $[18,19]$.

Considering the shortage and inconsistency of the existing empirical evidence, we conducted this exploratory study to quantitatively evaluate the impact of " $4+7$ " policy on the volume, expenditures, and daily cost of antihypertensive drugs.

\section{Methods}

\section{The policy intervention}

In China, the NCDP policy was initiated and organized by the National Healthcare Security Administration (NHSA) of the PRC. The highlight of this policy lies in the implementation of "volume-based procurement", in which "Trade-for-price" and "Guarantee of use" were taken as the core policy measures [20]. Table 1 illustrates the main timeline of " $4+7$ " policy.

Table 1

Timeline for " $4+7$ " policy.

\begin{tabular}{|ll|}
\hline Date & Key events \\
\hline $\begin{array}{l}\text { June 20, } \\
2018\end{array}$ & $\begin{array}{l}\text { Premier Li Keqiang presided over a State Council executive meeting to discuss the work of "National Centraliz } \\
\text { Drug Procurement (NCDP) pilot". }\end{array}$ \\
$\begin{array}{ll}\text { November } \\
14,2018\end{array}$ & $\begin{array}{l}\text { President Xi Jinping presided over the fifth meeting of Commission for Deepening Overall Reform of the CPC } \\
\text { Central Committee. This meeting approved the Pilot Program of National Centralized Procurement Drug }\end{array}$ \\
\hline
\end{tabular}

November Shanghai Joint Procurement Office issued the $4+7$ City Centralized Drug Procurement Document, and 15, 2018 announced the procurement plan. A total of 31 drug varieties were involved.

December At 8:30 - 10:00 am, drug manufacturers submit quotation application and relevant materials.

6, 2018

December At 2:00 pm, carry out negotiations and bidding.

6, 2018

December Shanghai Joint Purchasing Office announced the proposed bidding results.

7, 2018

December Chinese government organized the deployment meeting for the pilot work of National Centralized Drug 7, 2018 Procurement and Use. Vice premier Sun Chunlan attended the meeting and delivered a speech, emphasized the importance of steady promoting NCDP pilot work.

December Shanghai Joint Purchasing Office announced the bidding results. 25 drugs (by generic name) in the " $4+7$ " List 17,2018 were included, with an average price cut of $52 \%$ and the highest price cut of $96 \%$.

January General Office of the State Council issued the Notice on Issuing the Pilot Program for National Centralized Drug 1, 2019 Procurement and Use (GBF [2019] No.2).

March to From March 1 to April 1, 2019, 11 pilot cities started the procurement of winning products one after another. April,

2019

(a) "Trade-for-price": Under the " $4+7$ " policy, each public medical institution (i.e. public hospital and government-run primary healthcare institution) in the 11 pilot cities was required to submit the annual agreed procurement volume of the " $4+7$ " List drugs to the NHSA of the PRC. The agreed procurement volume is the expected annual purchase volume of a certain drug (by generic name) estimated with reference to the use volume in the previous year. The NHSA of the PRC then organized competitive bidding 
and price negotiation based on the $60 \%-70 \%$ of the annual procurement volume of 11 pilot cities. Pharmaceutical manufacturers hold original branded drugs that beyond patent protection period and generic drugs that passed the consistency evaluation of quality and efficacy were eligible to participate in the bidding. The pharmaceutical manufacturer with the lowest bid price won the bid. On December 17, 2018, the Joint Procurement Office announced the list of the 25 bid-winning products (by manufacturer), as well as the bid-winning price [9].

(b) "Guarantee of use": 11 pilot cities were required to start implementing the bid winning results before April 1, 2019. The purchases of all the bid-winning products were carried out on the provincial drug bidding and procurement platform. Besides, the use volume of each bid-winning product in each public medical institution in pilot cities was monitored and assessed by the NHSA of the PRC to ensure the completion of the agreed procurement volume [21]. In most of the pilot cities, medical institutions conduct monthly assessments on relevant clinical departments to ensure the use of bid-winning products.

On March 28, 2019, Shenzhen implemented the " $4+7$ " biding results, starting to purchase the 25 bid-winning drugs at the winning bid prices [11].

\section{Data sources}

This study used data from the Centralized Drug Procurement Survey in Shenzhen 2019 (CDPS-SZ 2019). In China, the CDPS-SZ 2019 was organized and conducted by the Global Health Institute of Wuhan University between December 2019 and January 2020. The survey aimed to evaluate the effect of pharmaceutical policies in Shenzhen, and collected monthly drug purchase order data between 2017 and 2019. In the CDPS-SZ 2019 database, each purchase order record included purchase date, generic name, dosage form, specification, pharmaceutical manufacturer, price per unit, purchase volume, purchase expenditures, etc. A general database containing 963127 monthly aggregated purchase order records was established, involving 1079 drug varieties (by generic name), 346 medical institutions, 857 pharmaceutical manufacturers. The total purchase expenditures reached 20.9 billion RMB.

In China, under the zero-markup drug policy [22], the drug purchase prices in public medical institutions is the same as the prices used by patients. Since 2015, it was required that all drugs used by public medical institutions should be purchased through the provincial-level drug centralized procurement platform [23]. Therefore, in Shenzhen, the drug purchase data of public medical institutions in the CDPS-SZ 2019 database is generally consistent with the drug use data.

\section{Samples}

In this study, we extracted data from the CDPS-SZ 2019 database with the following criteria: (a) the drug scope was " $4+7$ " policyrelated antihypertensive drugs, including 7 antihypertensive drugs in the " $4+7$ " List [24] and the alternative antihypertensive drugs that have an alternative relationship with " $4+7$ " List drugs in clinical use (Supplementary table 1). The alternative antihypertensive drugs were determined based on the Monitoring Plan Work of National Centralized Drug Procurement and Use issued by the NHSA of the PRC [21]. The " $4+7$ " List drugs were then sorted into bid-winning products and non-winning products [9]. Bid-winning products referred to products that won the tender in " $4+7$ " policy, otherwise they were deemed to be non-winning products. (b) the time period was 24 months from January 2018 and December 2019. (c) the scope of medical institutions were all the public medical institutions in Shenzhen. In China, the public medical institution consists of public hospital and governmentrun community healthcare center.

Purchase order records with incomplete information were excluded. Finally, 18,115 purchase order records of 24 antihypertensive drugs (by generic name) were included in the analysis.

\section{Outcome measures}

Three outcome measures were included: volume, expenditures, and daily cost. Expenditure data was reported in CNY. Volume was measured using Defined Daily Doses (DDDs), a measurement developed by the World Health Organization (WHO) to compare drug consumptions [25]. In this study, the DDD value of each medication is determined according to the Guidelines for ATC classification and DDD assignment 2021 [26]. Daily cost of each group of drugs was assessed by Defined Daily Drug cost (DDDc), which was calculated as Expenditures/DDDs.

\section{Statistical analysis}

Page $4 / 15$ 
Descriptive statistics were used. We first described the change of the volume, expenditures, and DDDc of policy-related antihypertensive drugs in the corresponding period before (March to December 2018) and after (March to December 2019) the implementation of " $4+7$ " policy.

A single-group Interrupted Time Series (ITS) was designed to assess the change in volume, expenditures, and DDDc of associated with the implementation of " $4+7$ " policy. ITS is a commonly used approach for evaluating changes in longitudinal series following a quasi-experimental intervention occurring at a fixed point in time [27]. The date of implementing " $4+7$ " bid-winning results in Shenzhen (March 2019) was regarded as the intervention time point for ITS analyses. We used segmented regression models that control for baseline trends to estimate changes in the levels and trends of each outcome variable after " $4+7$ " policy. The following model was developed [28]:

$Y_{t}=\beta_{0}+\beta_{1} \times$ time $_{t}+\beta_{2} \times$ intervention $_{t}+\beta_{3} \times$ time after intervention $_{t}+\varepsilon_{t}$

$Y_{t}$ is the outcome variable (volume, expenditures, and DDDc). $\beta_{0}$ estimates the baseline level of the outcome variable at the beginning of the observation period. $\beta_{7}$ estimates the slope prior to intervention. $\beta_{2}$ estimates the change in level in the period immediately following policy intervention. $\beta_{3}$ estimates the differences between pre-and post-intervention slopes. $\varepsilon_{t}$ is an estimate of the random error at time $t$. The relative change in level of each outcome variable after " $4+7$ " policy was expressed as $\beta_{2} / \beta_{0}$ [29]. We used Cumby-Huizinga test to detect the autocorrelation of each model [30]. If auto-correlation is detected, the PraisWinsten method was applied to estimate the regression [31]. The Durbin-Watson test was performed to indicate the model's adjustment effect on autocorrelation, a Durbin-Watson $d$ value of around 2 indicates no sign of auto-correlation [32]. All analyses were performed using Stata version 16.0.

\section{Results}

\section{Descriptive analysis}

A total of 24 antihypertensive drugs (by generic name) purchased between January 2018 and December 2019 were included in this study. Among them, 7 were " $4+7$ " List drugs and 17 were alternative drugs. A total of 36 pharmaceutical manufactures and 71 public medical institutions were involved. The total purchase volume and expenditures of included antihypertensive drugs were 212.03 million DDD and 545.92 million CNY, respectively.

\section{Completion rate of bid-winning antihypertensive drugs}

Based on the annual agreed purchase volume of each bid-winning drug submitted by each pilot city [24], six of the seven bidwinning antihypertensive drugs were submitted annual agreed purchase volume in Shenzhen: Irbesartan (4.71 million DDD), Amlodipine (12.06 million DDD), Fosinopril (0.74 million DDD), Irbesartan hydrochlorothiazide (0.50 million DDD), Losartan (2.47 million DDD), and Enalapril (1.59 million DDD). We calculated the completion rate of the 6 antihypertensive drugs in Shenzhen during the nine months of policy implementation from April 1 to December 31, 2019 (Fig. 1). The overall completion rate of $173.42 \%$ was observed for the six " $4+7$ " List antihypertensive drugs. Except for Fosinopril (77.74\%), the annual purchase volume of the five antihypertensive drugs was completed ahead of schedule within nine months. The completion rate of Irbesartan hydrochlorothiazide even reached $616.72 \%$.

\section{The change of volume, expenditures, and DDDc}

Table 2 shows the changes in volume, expenditures, and DDDc of policy-related antihypertensive drugs in the corresponding period before (March to December 2018) and after (March to December 2019) the implementation of "4 + 7" policy. After policy intervention, the bid-winning antihypertensive drugs increased markedly in the volume (1268.54\%) and expenditures (206.42\%), and decreased in the DDDc (77.61\%). Non-winning antihypertensive drugs reduced by $73.11 \%$ and $70.93 \%$ in the volume and expenditures, and increased by $8.11 \%$ in the DDDc. As for the alternative drugs that have an alternative relationship with " $4+7$ " List drugs in clinical use, the volume and expenditures increased by $25.04 \%$ and $23.12 \%$, and the DDDc slightly dropped by $1.53 \%$. 
In terms of the overall policy-related antihypertensive drugs included in this study, the volume raised by $47.63 \%$, the expenditures and DDDc decreased by $0.63 \%$ and $32.69 \%$.

Table 2

The change of volume, expenditures, and DDDc of policy-related antihypertensive drugs in the pre- and post-" $4+7$ " periods.

\begin{tabular}{|lllllllllll|l|}
\hline & \multicolumn{3}{c}{ Volume (thousand DDD) } & \multicolumn{2}{c}{ Expenditures (thousand CNY) } & \multicolumn{3}{c|}{ DDDc (CNY) } \\
\hline & Pre- & Post- & GR $(\%)$ & Pre- & Post- & GR (\%) & Pre- & Post- & GR (\%) \\
\hline "4 + 7" List drugs & 24.53 & 47.13 & 92.15 & 73.81 & 37.44 & -49.28 & 3.01 & 0.79 & -73.60 \\
\hline Bid-winning drugs & 3.02 & 41.35 & 1268.54 & 5.76 & 17.66 & 206.42 & 1.91 & 0.43 & -77.61 \\
\hline Non-winning drugs & 21.51 & 5.78 & -73.11 & 68.05 & 19.78 & -70.93 & 3.16 & 3.42 & 8.11 \\
\hline Alternative drugs & 48.36 & 60.46 & 25.04 & 151.16 & 186.11 & 23.12 & 3.13 & 3.08 & -1.53 \\
\hline Overall policy-related & 72.89 & 107.60 & 47.63 & 224.97 & 223.55 & -0.63 & 3.09 & 2.08 & -32.69 \\
\hline
\end{tabular}

DDD, Daily Defined Dose; CNY, Chinese Yuan; DDDc, Defined Daily Drug cost; GR, Growth Rate. Pre- refers to March to December 2018; Post- refers to March to December 2019.

Table 3 illustrates the changes of each of the seven " $4+7$ " List drug. After the implementation of " $4+7$ " policy, 7 antihypertensive drugs in the " 4 + 7" List increased by 22.60 million DDD (92.15\%) in the volume, and decreased by 36.37 million CNY (-49.28\%) and 2.22 CNY (-73.60\%) in the expenditures and DDDc, respectively. The volume of 7 drugs all increased compared with the pre-"4 +7 " period, with the growth rate ranging from $14.69-123.10 \%$. Five of the 7 drugs decreased in the expenditures after " $4+7$ " policy, with the reduction ranging from $16.47-60.95 \%$. As for the DDDc of " $4+7$ " List antihypertensive drugs, Enalapril increased by $42.61 \%$, and five drugs decreased between $54.81 \%$ and $82.50 \%$.

Table 3

The change of the volume, expenditures, and DDDc of each " $4+7$ " List antihypertensive drug in the pre- and post-" $4+7$ " periods.

\begin{tabular}{|c|c|c|c|c|c|c|c|c|c|}
\hline & \multicolumn{3}{|c|}{ Volume (million DDD) } & \multicolumn{3}{|c|}{ Expenditures (million CNY) } & \multicolumn{3}{|c|}{ DDDc (CNY) } \\
\hline & Pre- & Post- & $\mathrm{GR}(\%)$ & Pre- & Post- & GR (\%) & Pre- & Post- & $\begin{array}{l}\text { GR } \\
(\%)\end{array}$ \\
\hline Amlodipine & 11790.55 & 26305.12 & 123.10 & 39505.03 & 15424.75 & -60.95 & 3.35 & 0.59 & -82.50 \\
\hline Irbesartan & 6017.09 & 9736.77 & 61.82 & 15173.01 & 7709.53 & -49.19 & 2.52 & 0.79 & -68.60 \\
\hline $\begin{array}{l}\text { Irbesartan } \\
\text { hydrochlorothiazide }\end{array}$ & 1909.19 & 3899.69 & 104.26 & 6639.64 & 5545.92 & -16.47 & 3.48 & 1.42 & -59.11 \\
\hline Fosinopril & 572.97 & 657.15 & 14.69 & 3081.37 & 1531.17 & -50.31 & 5.38 & 2.33 & -56.67 \\
\hline Lisinopril & 0.00 & 6.02 & - & 0.00 & 2.77 & - & - & 0.46 & - \\
\hline Losartan & 3339.10 & 4749.23 & 42.23 & 8872.47 & 5702.14 & -35.73 & 2.66 & 1.20 & -54.81 \\
\hline Enalapril & 900.38 & 1780.08 & 97.70 & 540.23 & 1523.16 & 181.95 & 0.60 & 0.86 & 42.61 \\
\hline Total & 24529.28 & 47134.07 & 92.15 & 73811.75 & 37439.44 & -49.28 & 3.01 & 0.79 & -73.60 \\
\hline
\end{tabular}

\section{ITS analysis}


The results of ITS analysis (Table 4) indicated that the volume of bid-winning antihypertensive drugs significantly increased by 1311.76\% (3.12 million DDD, 95\% $C l=2.14$ to $4.10, p<0.001)$ after " $4+7$ " policy. The volume of non-winning antihypertensive drugs significantly decreased by $140.29 \%$ (-2.33 million DDD, $95 \% C l=-2.83$ to $-1.82, p<0.001)$, and the slope significantly decreased in the post-intervention period ( -0.11 million DDD per month, $95 \% \mathrm{Cl}=-0.19$ to $-0.03, p<0.01)$. No significant differences were detected for the volume of " $4+7$ " List drugs, alternative drugs, and the overall policy-related antihypertensive drugs (all $p$ values $>0.05$ ). 
Table 4

Results of ITS analysis for the change of volume, expenditures, and DDDc of policy-related antihypertensive drugs.

\begin{tabular}{|c|c|c|c|c|c|c|}
\hline & \multicolumn{2}{|l|}{ Volume } & \multicolumn{2}{|c|}{ Expenditures } & \multicolumn{2}{|l|}{ DDDc } \\
\hline & Coef. & $95 \% \mathrm{Cl}$ & Coef. & $95 \% \mathrm{Cl}$ & Coef. & $95 \% \mathrm{Cl}$ \\
\hline \multicolumn{7}{|l|}{ Bid-winning drugs } \\
\hline Baseline change, $\beta_{0}$ & 0.24 & $(-0.35,0.83)$ & 0.47 & $(0.18,0.77)^{\star \star}$ & 2.04 & $(1.96,2.12)^{\star \star \star}$ \\
\hline Baseline trend, $\beta_{1}$ & 0.01 & $(-0.07,0.09)$ & 0.02 & $(-0.02,0.06)$ & -0.02 & $(-0.03,-0.01)^{\star \star}$ \\
\hline Level change, $\beta_{2}$ & 3.12 & $(2.14,4.10)^{\star \star \star}$ & 0.93 & $(0.45,1.42)^{\star \star}$ & -1.30 & $(-1.43,-1.18)^{\star \star \star}$ \\
\hline Trend change, $\beta_{3}$ & 0.13 & $(-0.02,0.28)$ & 0.01 & $(-0.06,0.09)$ & 0.01 & $(-0.01,0.03)$ \\
\hline$R^{2}$ & 0.94 & & 0.85 & & 0.99 & \\
\hline Durbin-Watson $d$ & 2.12 & & 2.11 & & 1.91 & \\
\hline $\mathrm{RC}(\%)$ & 1311.76 & & 197.05 & & -63.79 & \\
\hline \multicolumn{7}{|l|}{ Non-winning drugs } \\
\hline Baseline change, $\beta_{0}$ & 1.66 & $(1.36,1.96)^{\star \star \star}$ & 5.39 & $(4.40,6.38)^{\star \star \star}$ & 3.20 & $(3.09,3.30)^{\star \star \star}$ \\
\hline Baseline trend, $\beta_{1}$ & 0.09 & $(0.05,0.13)^{\star \star \star}$ & 0.26 & $(0.13,0.39)^{\star \star \star}$ & -0.004 & $(-0.02,0.01)$ \\
\hline Level change, $\beta_{2}$ & -2.33 & $(-2.83,-1.82)^{\star \star \star}$ & -6.92 & $(-8.57,-5.28)^{\star \star \star}$ & 0.28 & $(0.11,0.46)^{\star \star}$ \\
\hline Trend change, $\beta_{3}$ & -0.11 & $(-0.19,-0.03)^{\star \star}$ & -0.34 & $(-0.59,-0.09)^{\star}$ & 0.01 & $(-0.02,0.03)$ \\
\hline$R^{2}$ & 0.92 & & 0.91 & & 0.65 & \\
\hline Durbin-Watson $d$ & 2.20 & & 2.11 & & 2.00 & \\
\hline $\mathrm{RC}(\%)$ & -140.29 & & -128.42 & & 8.82 & \\
\hline \multicolumn{7}{|l|}{ “4 + 7" List drugs } \\
\hline Baseline change, $\beta_{0}$ & 1.97 & $(1.19,2.74)^{\star \star \star}$ & 5.88 & $(4.73,7.02)^{\star \star \star}$ & 3.06 & $(2.89,3.24)^{\star \star \star}$ \\
\hline Baseline trend, $\beta_{1}$ & 0.09 & $(-0.01,0.19)$ & 0.28 & $(0.13,0.43)^{\star \star}$ & -0.01 & $(-0.03,0.02)$ \\
\hline Level change, $\beta_{2}$ & 0.95 & $(-0.33,2.23)$ & -5.96 & $(-7.87,-4.04)^{\star \star \star}$ & -1.94 & $(-2.21,-1.66)^{\star \star \star}$ \\
\hline Trend change, $\beta_{3}$ & 0.02 & $(-0.18,0.22)$ & -0.33 & $(-0.62,-0.04)^{\star}$ & -0.04 & $(-0.09,0.002)$ \\
\hline$R^{2}$ & 0.74 & & 0.84 & & 0.98 & \\
\hline Durbin-Watson $d$ & 2.15 & & 2.15 & & 1.96 & \\
\hline $\mathrm{RC}(\%)$ & 48.27 & & -101.34 & & -63.24 & \\
\hline \multicolumn{7}{|l|}{ Alternative drugs } \\
\hline Baseline change, $\beta_{0}$ & 3.91 & $(3.11,4.71)^{\star \star \star}$ & 12.76 & $(10.36,15.17)^{\star \star \star}$ & 3.24 & $(3.18,3.31)^{\star \star \star}$ \\
\hline Baseline trend, $\beta_{1}$ & 0.16 & $(0.05,0.26)^{\star \star}$ & 0.41 & $(0.10,0.73)^{\star}$ & -0.02 & $(-0.02,-0.01) \star \star$ \\
\hline Level change, $\beta_{2}$ & -0.91 & $(-2.24,0.42)$ & -1.99 & $(-5.99,2.02)$ & 0.14 & $(0.03,0.25)^{\star}$ \\
\hline
\end{tabular}

DDDc, Defined Daily Drug cost; $C l$, confidence interval; $R C$, relative change. 


\begin{tabular}{|c|c|c|c|c|c|c|}
\hline \multirow[b]{2}{*}{ Trend change, $\beta_{3}$} & \multicolumn{2}{|c|}{ Volume } & \multicolumn{2}{|c|}{ Expenditures } & \multicolumn{2}{|l|}{ DDDc } \\
\hline & 0.01 & $(-0.20,0.21)$ & -0.01 & $(-0.62,0.60)$ & 0.00 & $(-0.02,0.01)$ \\
\hline$R^{2}$ & 0.62 & & 0.56 & & 0.59 & \\
\hline Durbin-Watson $d$ & 2.25 & & 2.18 & & 2.03 & \\
\hline $\mathrm{RC}(\%)$ & -23.23 & & -15.57 & & 4.26 & \\
\hline \multicolumn{7}{|c|}{$\begin{array}{l}\text { Overall policy-related } \\
\text { antihypertensive drugs }\end{array}$} \\
\hline Baseline change, $\beta_{0}$ & 5.85 & $(4.49,7.20)^{\star \star \star}$ & 18.59 & $(15.28,21.90)^{\star \star \star}$ & 3.18 & $(3.09,3.27)^{\star \star \star}$ \\
\hline Baseline trend, $\beta_{1}$ & 0.25 & $(0.07,0.43)^{\star \star}$ & 0.70 & $(0.26,1.13)^{\star \star}$ & -0.01 & $(-0.02,0.00)^{*}$ \\
\hline Level change, $\beta_{2}$ & -0.01 & $(-2.26,2.25)$ & -8.02 & $(-13.54,-2.51)^{\star \star}$ & -0.78 & $(-0.92,-0.63)^{\star \star \star}$ \\
\hline Trend change, $\beta_{3}$ & 0.02 & $(-0.33,0.36)$ & -0.35 & $(-1.19,0.49)$ & -0.02 & $(-0.04,0.004)$ \\
\hline$R^{2}$ & 0.74 & & 0.47 & & 0.98 & \\
\hline Durbin-Watson $d$ & 2.36 & & 2.24 & & 2.35 & \\
\hline $\mathrm{RC}(\%)$ & -0.09 & & -43.17 & & -24.38 & \\
\hline
\end{tabular}

ITS analysis indicated that the expenditures of bid-winning antihypertensive drugs significantly increased by $197.05 \%$ ( 0.93 million $\mathrm{CNY}, 95 \% \mathrm{Cl}=0.45$ to $1.42, p<0.01$ ) after " $4+7$ " policy. The expenditures of non-winning antihypertensive drugs significantly decreased by $128.42 \%$ ( -6.92 million CNY, $95 \% C l=-8.57$ to $-5.28, p<0.001)$, and the slope significantly declined in the post-intervention period ( -0.34 million CNY per month, $95 \% \mathrm{Cl}=-0.59$ to $-0.09, p<0.05)$. The expenditures of " $4+7$ " List antihypertensive drugs significantly decreased by $101.34 \%$ ( -5.96 million CNY, $95 \% C l=-7.87$ to $-4.04, p<0.001)$, and the slope significantly decreased in the post-intervention period ( -0.33 million CNY per month, $95 \% \mathrm{Cl}=-0.62$ to $-0.04, p<0.05)$. No significant difference was observed for the alternative drugs neither in expenditure level change nor in expenditure trend change (all $p$-values > 0.05). After the implementation of " $4+7$ " policy, the expenditures of the overall policy-related antihypertensive drugs significantly decreased by $43.17 \%$ (-8.02 million CNY, $95 \% \mathrm{Cl}=-13.54$ to $-2.51, p<0.01)$.

After policy intervention, the prominent decline of $63.79 \%, 63.24 \%$, and $24.38 \%$ were observed in the DDDc of bid-winning antihypertensive drugs(-1.30 CNY, $95 \% C l=-1.43$ to $-1.18, p<0.001)$, " $4+7$ " List antihypertensive drugs $(-1.94 \mathrm{CNY}, 95 \% \mathrm{Cl}=-2.21$ to $-1.66, p<0.001)$, and the overall policy-related antihypertensive drugs $(-0.78 \mathrm{CNY}, 95 \% \mathrm{Cl}=-0.92$ to $-0.63, p<0.001)$. The DDDc of non-winning antihypertensive drugs $(0.28 \mathrm{CNY}, 95 \% \mathrm{Cl}=0.11$ to $0.46, p<0.01)$ and alternative drugs $(0.14 \mathrm{CNY}, 95 \% \mathrm{Cl}=0.03$ to $0.25, \mathrm{p}<0.05)$ significantly increased by $8.82 \%$ and $4.26 \%$.

\section{Discussion}

In the present study, we analyzed the impact of the " $4+7$ " policy on the volume, expenditures, and DDDc of policy-related antihypertensive drugs. By calculating the completion rate, we found that the implementation of " $4+7$ " policy in Shenzhen is generally progressing well. When the policy is implemented for nine months, the overall purchase volume of bid-winning antihypertensive drugs in Shenzhen has reached up to $173.42 \%$ of the initially committed annual procurement volume. This is in accordance with the implementation progress of $183 \%$ in 11 pilot cities announced by the NHSA of the PRC in December 2019 [33]. 
After the implementation of " $4+7$ " policy, the DDDc of bid-winning antihypertensive drugs decreased remarkably, as well as the " 4 +7 " List drugs and the overall policy-related antihypertensive drugs. However, the ITS analysis indicated a prominent increase in the DDDc of non-winning antihypertensive drugs and the alternative antihypertensive drugs. Previous research reported the opposite results of this study that the price of non-winning drugs decreased after the implementation of " $4+7$ " policy $[16,17]$, which fits well the cross-price elasticity theory $[34,35]$. Thus, under " $4+7$ " policy, the price changes in the antihypertensive drug category might have specific characteristics. We supposed there is certain possibility that the price of drugs without policy intervention have increased after policy implementation. The increasing of DDDc for the non-winning and the alternative drugs might be related to the unreasonable prescription behavior, such as increased daily doses of some antihypertensive drugs [36, 37]. In the future, it is recommended to strengthen the price monitoring and prescription management of NCDP policy-related drugs.

Under NCDP policy, the original drugs and generic drugs that had passed the consistency evaluation of quality and efficacy were eligible for the national level volume-based procurement. In this study, we found that the consumption of bid-winning antihypertensive drugs raised remarkably (1268.54\%), even led to the large increase in the use volume of drugs with the same generic name (92.15\%). This fits with Yang et al.'s finding [38] on SSRI antidepressants and Sheng et al.'s study [39] on nucleoside anti-hepatitis B virus drugs. However, we found that there were significant differences in the changes of purchase volume for different drugs (by generic name) before and after policy intervention, and the growth rate fluctuated between $14.69 \%$ and $123.10 \%$. Previous studies mention that the poor antihypertensive efficacy and increased adverse reactions was observed in some bid-winning antihypertensive drugs $[19,18]$. In the future, relevant investigation at the patient-level might be necessary to deeply specifically understand the therapeutic effect and patient satisfaction of each drug in the NCDP List.

After the implementation of " $4+7$ " policy, the costs of the seven " $4+7$ " List antihypertensive drugs prominently decreased $101.34 \%$ (36.37 million CNY) compared with the pre-" 4 + 7" period, as well as the overall policy-related antihypertensive drugs. It indicated that there is a initiatory cost-saving effect of " $4+7$ " policy on the category of antihypertensive drugs. Price is the primary determinant of drug affordability [40], the unreasonable price and high expenditures of antihypertensive drugs have been an important factor restricted the effective control of drug burden of hypertensive patients in China [41]. Gheorghe et al. [42] reported that the annual medical expenditures per capita of hypertensive patients in low- and middle-income countries exceeds 5.9 times total medical expenditures per capita. Wang et al.'s [43] survey in Gansu, China indicated that the average monthly drug expenditures of hypertensive patients accounted for $16.4 \%$ of the total family expenses. Under the circumstance, " $4+7$ " policy has achieved significant cost-saving effects in the short term by including 7 antihypertensive drugs. In the future, more commonly used drugs such as medication for chronic diseases or drugs with large demand will be included in the volume-based procurement catalog, which is expected to effectively reduce the drug burden of patients in China.

\section{Limitations}

Several potential limitations should be mentioned regarding the present study. First, " $4+7$ " policy was implemented in 11 pilot cities, while limited by the data accessibility, this study only involved one pilot city (Shenzhen) for analysis. Second, the results of this study were based on drug purchase data, rather than drug use data (such as prescriptions). Although there is strong consistency between purchase data and use data under a series of policies. There is still a possibility that the two data sources may not exactly match, so there are certain limitations. Third, this study constructed ITS models using the data of 24 months and only 10 time points after policy intervention were involved, which may have certain limitations in exploring the long-term trend of " $4+7$ " policy.

\section{Conclusion}

This study assessed the impact of " $4+7$ " policy on policy-related hypertensive drugs in Shenzhen, China. An overall satisfying implementation effect was observed, for the purchase volume of bid-winning antihypertensive drugs has reached up to $173.42 \%$ of the initially committed annual procurement volume when the policy is implemented for nine months. The consumption of bidwinning antihypertensive drugs raised remarkably (1268.54\%) in Shenzhen, even led to the large increase in the use volume of drugs with the same generic name (92.15\%). After the implementation of " $4+7$ " policy, the DDDc of bid-winning antihypertensive drugs significantly decreased by $63.79 \%$, the overall costs of the 7 antihypertensive drugs in the " $4+7$ " List declined prominently (101.34\%). A preliminary positive policy effect of price cut and cost-saving was observed in the antihypertensive drug category.

Page $10 / 15$ 
However, the DDDc of non-winning hypertensive drugs and alternative drugs increased after policy intervention, suggesting that the price monitoring and drug use management regarding NCDP policy-related drugs should be strengthened in the future.

\section{Abbreviations}

CNY: Chinese Yuan; OECD: Organization for Economic Co-operation and Development; PRC: People's Republic of China; NCDP: National Centralized Drug Procurement; NHSA: National Healthcare Security Administration; CDPS-SZ 2019: Centralized Drug Procurement Survey in Shenzhen 2019; DDDs: Defined Daily Doses; WHO: World Health Organization; DDDc: Defined Daily Drug cost; ITS: Interrupted Time Series.

\section{Declarations}

\section{Ethics approval and consent to participate}

Not applicable. No ethical approval was required for this study by the authors' institution, because this manuscript does not involve the use of any animal or human data or tissue.

\section{Consent for publication}

Not applicable.

\section{Funding}

This work was supported by the Global Health Institute, Wuhan University, China. The role of funding body included designing the study, analysis and interpretation of data, and writing the manuscript.

\section{Availability of data and materials}

The datasets used and/or analyzed during the current study are available from the corresponding author on reasonable request.

\section{Authors' contributions}

YY and ZM designed the study. RT, SY, LM, and SH collected data. YY, LM, LX, and SH analyzed and interpreted data. YY, RT, SY, and LX drafted the first version of the manuscript. YY, RT, SY, LX, and ZM revised the manuscript. All authors have read and agreed to the published version of the manuscript.

\section{Competing interests}

The authors declare that they have no competing interests.

\section{References}

1 National Health Commission. 2018 Statistical Bulletin of China's Health Development. 2020. http://www.gov.cn/guoqing/2020-04/29/content_5507528.htm. Accessed March 20, 2021.

2 The General Office of the State Council. Notice of the General Office of the State Council on Issuing the Pilot Program for the Centralized Procurement and Use of Drugs Organized by the State. 2019. http://www.gov.cn/zhengce/content/201901/17/content_5358604.htm. Accessed March 18, 2021.

3 Mao Z, Yang Y, Chen L. Reform of Drug Supply and Guarantee System in China: Policy Measures and Effects. In: Wang C, Liang W, editors. Development Report on Health Reform in China (2020). Beijing: Social Sciences Academic Press; 2020. p. 96123.

4 Li Y, Yang L, Wang L, Zhang M, Huang Z, Deng Q et al. Burden of hypertension in China: A nationally representative survey of 174621 adults. INT J CARDIOL. 2016;227:516-23. doi: 10.1016/j.ijcard.2016.10.110. 
5 Chinese Pharmaceutical Association. Hospital Drug Monitoring Report 2019. 2020. https://www.cpa.org.cn//? do=info\&cid=75104. Accessed March 10, 2021.

6 Zhang W, Xu W, Yang X. Drug burden of antihypertensive drugs in 87 county level hospitals in China. Chongqing Medicine. 2020;49(19):3303-5. doi: 10.3969/j.issn.1671-8348.2020.19.035.

$7 \mathrm{Xu}$ Y, He J, Chen M, Song J, Jin C. International Experience of Centralized Drug Procurement and Enlightenment to China's National Level Volume-based Drug Procurement. Chinese Health Economics. 2021;40(04):93-6

8 National Health Development Research Center of the National Health Commission. 2018 China Total Health Expenditure Research Report. 2018.

9 Joint Procurement Office. Announcement of the results of the planned selection of centralized drug procurement in $4+7$ cities. 2018. http://www.smpaa.cn/gjsdcg/2018/12/07/8531.shtml. Accessed March 22, 2021.

10 Ye S, Kang Q, Gao J, Chen Q, Hu S, Cai H et al. Study Of Drug Price Index in Evaluating Implementation Effect of "4+7" Drug Centralized Procurement Policy in Fujian. China Health Insurance. 2020(08):35-9. doi: 10.19546/j.issn.1674-3830.2020.8.008.

11 Shenzhen Healthcare Security Bureau. Pilot Analysis on Drug Centralized Procurement and Use Organized by the State in Shenzhen. China Health Insurance. 2019(08):32-7. doi: 10.19546/j.issn.1674-3830.2019.8.008.

12 Huang D, Li Z, Zhao Q. Practice and Consideration of Drug Centralized Procurement and Use in Chengdu. China Health Insurance. 2019(08):20-3. doi: 10.19546/j.issn.1674-3830.2019.8.005.

13 Mu A. Preliminary Analysis on the Pilot Project of Drug Centralized Procurement and Use in Chongqing. China Health Insurance. 2019(08):24-7. doi: 10.19546/j.issn.1674-3830.2019.8.006.

14 Meng L. Analysis on the Pilot Operation of Drug Centralized Procurement and Use in Shenyang. China Health Insurance. 2019(08):28-31. doi: 10.19546/j.issn.1674-3830.2019.8.007.

15 Chen H, Liu Y, Yu X, Zhang D, Zhou H, Feng W et al. Data Analysis on Pilot Reform of Drug Centralized Procurement and Use --Taking Beijing Chaoyang Hospital as an Example. China Health Insurance. 2019(08):51-3. doi: 10.19546/j.issn.16743830.2019.8.012.

16 Wang N, Yang Y, Xu L, Mao Z, Cui D. Influence of Chinese National Centralized Drug Procurement on the price of policyrelated drugs: an interrupted time series

analysis. Preprints. 2021(2021040157). doi: 10.20944/preprints202104.0157.v1.

17 Wang H, Li X, Chen J. Impact of "4+7" City Drug Centralized Procurement Program on the utilization of original and generic cardiovascular drugs in a tertiary hospital. Journal of Pharmaceutical Practice. 2020;38(04):3738. doi: 10.12206/j.issn.1006-0111.202001054.

18 Yang Q, Gu H. Investigation of the implementation status of national centralized drug procurement in community health service centers of Shanghai. Shanghai Medical \& Pharmaceutical Journal. 2020;41(04):11-4

19 He J, Tang M, Cong L, Xu Y, Song J, Chen M et al. The impact of National Centralized Drug Procurement on the clinical management and drug use. Chinese Health Resources. 2021:1-3. doi: 10.13688/j.cnki.chr.2021.200802.

20 General Office of the State Council of the PRC. Pilot Program for National Centralized Drug Procurement and Use. 2019. http://www.gov.cn/zhengce/content/2019-01/17/content_5358604.htm. Accessed March 20, 2021.

21 Wang Y, Wu Z. Keypoint Analysis of Monitoring Plan for Centralized Drug Purchase and Use Project Pilot Launched Organized by State. China Pharmacy. 2019;30(17):2317-22. doi: 10.6039/j.issn.1001-0408.2019.17.03. 
22 Deng J, Tian H, Guo Y, Ma T, Sun Y, Zhang S et al. A retrospective and prospective assessment of the zero-markup drug reform in China from the perspective of policy diffusion. Int J Health Plann Manage. 2018;33(4):e918-29. doi: 10.1002/hpm.2562.

23 General Office of the State Council. Guiding Opinions on Improving the Centralized Drug Procurement in Public Hospitals (Guobanfa [2015] No. 7). 2015. http://www.gov.cn/zhengce/content/2015-02/28/content_9502.htm. Accessed March $20,2021$.

24 Joint Procurement Office. 4+7 City Drug Centralized Procurement Documents (No. GY-YD2018-1). 2018.

http://www.smpaa.cn/gjsdcg/2018/11/15/8511.shtml. Accessed March 20, 2021.

25 WHO Collaborating Centre for Drug Statistics Methodology. ATC/DDD Index 2021. 2020.

https://www.whocc.no/atc_ddd_index/. Accessed March 20, 2021.

26 WHO Collaborating Centre for Drug Statistics Methodology. Guidelines for ATC classification and DDD assignment 2021, Norway. 2021. http://www.whocc.no. Accessed March 20, 2021.

27 Lagarde M. How to do (or not to do) ... Assessing the impact of a policy change with routine longitudinal data. HEALTH POLICY PLANN. 2011;27(1):76-83. doi: 10.1093/heapol/czr004.

28 Wagner AK, Soumerai SB, Zhang F, Ross-Degnan D. Segmented regression analysis of interrupted time series studies in medication use research. J CLIN PHARM THER. 2002;27(4):299-309. doi: 10.1046/j.1365-2710.2002.00430.x.

29 Zhang F, Wagner AK, Soumerai SB, Ross-Degnan D. Methods for estimating confidence intervals in interrupted time series analyses of health interventions. J CLIN EPIDEMIOL. 2009;62(2):143-8. doi: 10.1016/j.jclinepi.2008.08.007.

30 Baum CF, Schaffer ME. ACTEST: Stata module to perform Cumby-Huizinga general test for autocorrelation in time series. 2013. https://ideas.repec.org/c/boc/bocode/s457668.html.

31 Linden A. Conducting interrupted time-series analysis for single- and multiple-group comparisons. The Stata Journal. 2015;15(2):480-500

32 Durbin J, Watson GS. Testing for serial correlation in least squares regression. I. BIOMETRIKA. 1950;37(3-4):409-28

33 National Healthcare Security Administration of the People's Republic of China. Answer to Reporters' Request about the Second Round of National Centralized Drug Procurement and Use. 2020.

http://www.nhsa.gov.cn/art/2020/1/17/art_38_2264.html.

34 Hursh SR. Behavioral economics of drug self-administration: an introduction. DRUG ALCOHOL DEPEN. 1993;33(2):165-72. doi: https://doi.org/10.1016/0376-8716(93)90058-X.

35 Hursh S, Bauman R. The behavioral analysis of demand. In: Green L, Kagel J, editors. Advances in behavioral economics: Ablex, Norwood, NJ; 1987. p. 117-65.

36 Yang Y, Chen L, Ke X, Mao Z, Zheng B. The impacts of Chinese drug volume-based procurement policy on the use of policyrelated antibiotic drugs in Shenzhen, 2018-2019: an interrupted time-series analysis. BMC Health Services Research (Preprints). 2021. doi: $10.21203 /$ rs.3.rs-143454/v1.

37 Yu C. The practical effects and system concerns of "4+7" drug procurement. Journal of Southwest Minzu University (Humanities and Social Science). 2020;41(04):34-9

38 Yang Q, Zang Y, Zhuang H, Guo W. Effects of "4+7" procurement with target quantity on the using of antidepressants in a hospital. Chinese Journal of Hospital Pharmacy. 2020;40(13):1479-83. doi: 10.13286/j.1001-5213.2020.13.16.

39 Sheng L, Li X, Hu X. Application of Nucleoside Anti-hepatitis B Drugs Based on "4+7" Quantified Purchasing. China Journal of Pharmaceutical Economics. 2019;14(07):36-9. doi: 10.12010/j.issn.1673-5846.2019.07.007. 
40 National Academies of Sciences EAM, Division HAM, Services BOHC, Therapies COEP. Making Medicines Affordable: A National Imperative. Washington (DC): National Academies Press (US); 2017.

$41 \mathrm{Li} \mathrm{H}$, Zhu J, CHhen Y. Usage of Original and Generic Antihypertensive Drugs in a Special Outpatient Department of a Hospital. China Pharmaceuticals. 2019;28(10):90-3. doi: 10.3969/j.issn.1006-4931.2019.10.030.

42 Gheorghe A, Griffiths U, Murphy A, Legido-Quigley H, Lamptey P, Perel P. The economic burden of cardiovascular disease and hypertension in low- and middle-income countries: a systematic review. BMC PUBLIC HEALTH. 2018;18(1):975. doi:

10.1186/s12889-018-5806-x.

43 Wang X, Zhang M, Zhao S, Lv M, Li Q, He L et al. Investigation on Household Medication-Taking Behavior and Affordability Among Patients Under Chronic Condition: A Survey Study in Gansu Province, China. FRONT PHARMACOL. 2020;11:1280. doi: 10.3389/fphar.2020.01280.

\section{Figures}

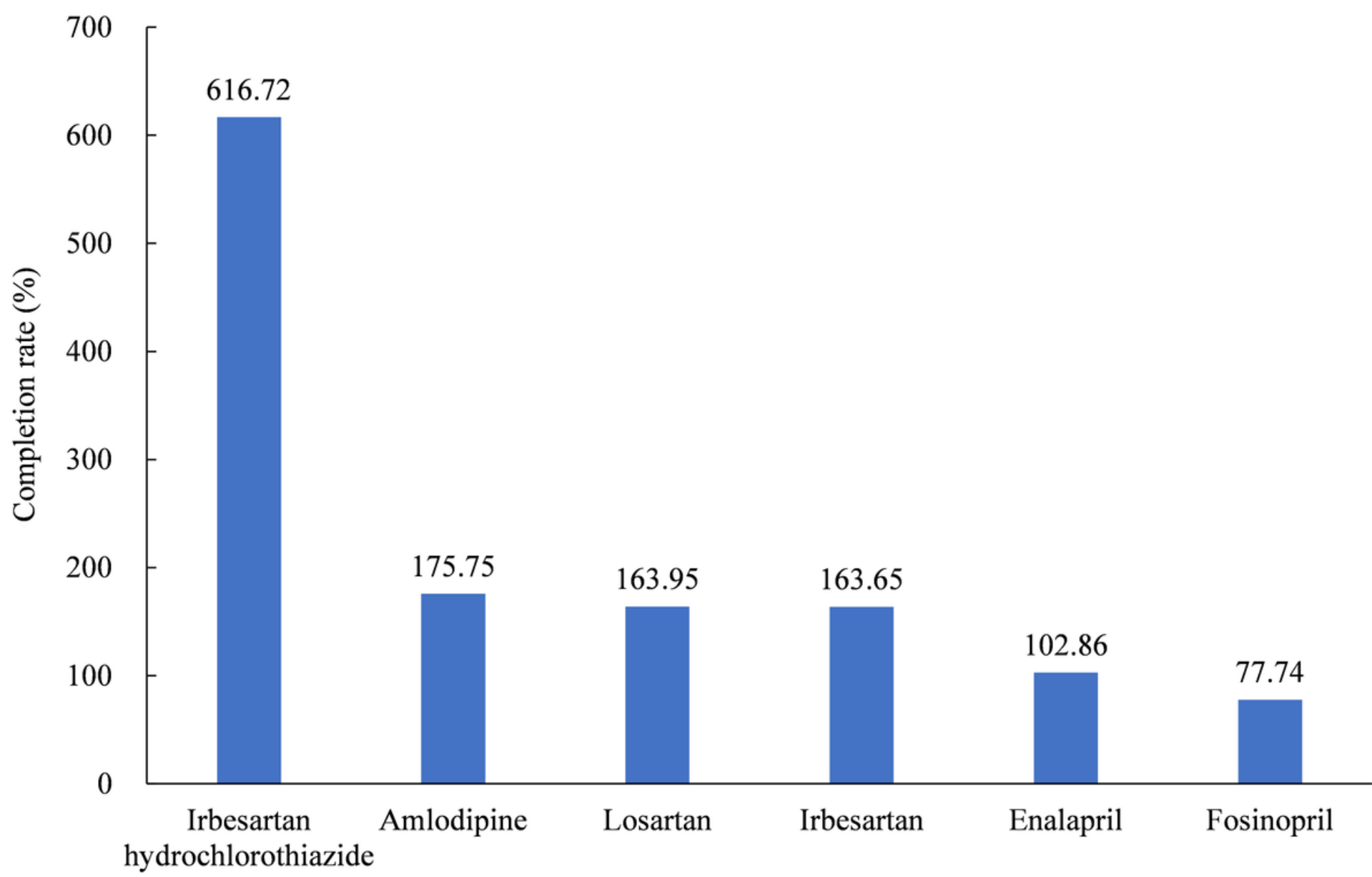

Figure 1

Completion rate of the agreed purchase volume of each bid-winning products in Shenzhen during April to December 2019. Note: Shenzhen did not submit the agreed purchase volume of Lisinopril, thus only 6 drugs were shown in this figure.

\section{Supplementary Files}

This is a list of supplementary files associated with this preprint. Click to download. 
- Tables.docx

- file.docx

- file.docx

Page 15/15 